\title{
Effect of nitrogen on deposition and field emission properties of boron-doped micro- and nano-crystalline diamond films
}

\author{
L. A. Li, S. H. Cheng, H. D. Li*, Q. Yu, J. W. Liu and X. Y. Lv
}

In this paper, we report the effect of nitrogen on the deposition and properties of boron doped diamond films synthesized by hot filament chemical vapor deposition. The diamond films consisting of micro-grains (nano-grains) were realized with low (high) boron source flow rate during the growth processes. The transition of micro-grains to nano-grains is speculated to be strongly (weekly) related with the boron (nitrogen) flow rate. The grain size and Raman spectral feature vary insignificantly as a function of the nitrogen introduction at a certain boron flow rate. The variation of electron field emission characteristics dependent on nitrogen is different between microcrystalline and nanocrystalline boron doped diamond samples, which are related to the combined phase composition, boron doping level and texture structure. There is an optimum nitrogen proportion to improve the field emission properties of the boron-doped films.

Keywords: Chemical vapor deposited diamond film; Nitrogen effect; Boron doping; Microcrystalline; Nanocrystalline; Electron field emission

Citation: L. A. Li, S. H. Cheng, H. D. Lị, Q. Yu, J. W. Liu and X. Y. Lv, "Effect of nitrogen on deposition and field emission properties of boron-doped micro- and nano-crystalline diamond films", Nano-Micro Lett. 2, 154 (2010). doi: 10.5101/nml.v2i3.p154-159.

Chemical vapor deposited (CVD) diamond films have attracted intensive interests owing to its outstanding properties, such as wide band gap, super-hardness, high thermal conductivity, high carrier mobility and high chemical inertness. However, it is somehow limited for electronic application of diamond due to its insulating property, unless the dopants are incorporated into diamond [1]. Various elements (e.g. nitrogen N, boron B, sulfur S, and phosphorus $\mathrm{P}$ ) have been introduced into diamond to adjust their electronic properties. The nucleation process [2], growth mechanism [3], impurity distribution [4] and properties [5-7] affected by doping have been extensively investigated. However, in previous work, the understanding on how the dopants (mainly refer to $\mathrm{N}$ and $\mathrm{B}$ ) modify the growth and properties of diamond films was mostly emphasized on the sole doping by boron or nitrogen. Recently, there are a few reports on the diamond deposition with co-dopants [8,9]. Hartmann et al. [8] reported that in highly boron-doped micro-diamond, large (small) additions of nitrogen would stabilize the diamond structure (induce more graphite formation). The electronic structure of boron-doped nano-diamond films can be markedly modified by nitrogen and there was an optimized gas proportion to achieve improved field emission [9]. However, the corresponding underlying mechanism is still not very clear. Furthermore, growth features related to the co-doping of boron and nitrogen are desirable to be investigated in detail.

In this letter, by hot filament CVD (HFCVD), the microcrystalline diamond (MCD) and nanocrystalline diamond (NCD) films with co-introducing boron and nitrogen were synthesized by adjusting the gas proportion of nitrogen and boron in the reaction ambient. The effect of nitrogen on the 
deposition, structural characteristics and electron field emission (EFE) properties of boron-doped diamond films has been systematically investigated.

The polycrystalline diamond films were synthesized by a HFCVD system [5]. The p-type Si (111) substrates were abraded by diamond paste and cleaned ultrasonically in ethanol solution for nucleation enhancement. A spiral tantalum wire was used as the filament, and its heating temperature was about $2200^{\circ} \mathrm{C}$. The total pressure was $4 \mathrm{kPa}$ with $2 \% \mathrm{CH}_{4}$ in the precursor gases of methane $\left(\mathrm{CH}_{4}\right.$, flow rate: $\left.4 \mathrm{sccm}\right)$ and hydrogen $\left(\mathrm{H}_{2}\right.$, flow rate: $200 \mathrm{sccm})$. The substrate temperature was approximately $800^{\circ} \mathrm{C}$ monitored by an optical pyrometer. The boron source was introduced by bubbling the $\mathrm{H}_{2}$ gas (flow rates: 2, $10 \mathrm{sccm}$ ) through the liquid trimethylborate $\left(\mathrm{B}\left(\mathrm{OCH}_{3}\right)_{3}\right)$ (ambient temperature kept at $25^{\circ} \mathrm{C}$ ), and the nitrogen gas flow rates were 1 , 4 , and $8 \mathrm{sccm}$ at different boron flow rates. For simplification, in this letter, the boron flow rate (determining the boron doping level) is expressed by the corresponding bubbling $\mathrm{H}_{2}$ flow rate. Six films were grown for 4 hours with the flow-rate ratios of $\mathrm{N}$ : $\mathrm{B}=1: 2,4: 2,8: 2,1: 10,4: 10$ and $8: 10$ in sccm, respectively, and the obtained films were labeled as sample (a) (f) in turn.

The morphologies, microstructure and phase composition of the as-grown diamond films were characterized by means of scanning electron microscope (SEM, JXA-8200), X-ray diffraction (XRD, Rigaku D/MAX-RA with $\mathrm{Cu} \mathrm{K} \alpha$ radiation), and Raman spectroscopy (Renishaw inVia Raman microscope with $514.5 \mathrm{~nm}$ line of $\mathrm{Ar}^{+}$laser). The electron field emission (EFE) measurements were performed in the chamber at a high vacuum of $10^{-7} \mathrm{~Pa}$.

Figure 1 shows the SEM images of the six diamond films (a) (f). The morphologies of samples (a), (b) and (c) deposited with $2 \mathrm{sccm}$ boron flow rate are similar, and the evident twin features are represented in the well (111) faceted grains. The average grain size is about $2 \mu \mathrm{m}$ for the samples, and the samples are named as MCD films. As the boron flow rate increases to 10 sccm, all samples (samples d, e, and f, named NCD films) show round shaped grains consisting of nano-sized crystals, and the morphologies are weekly dependent on the nitrogen flow rate. It worth pointing out that, there are many (100) faced grains with the size of about $500 \mathrm{~nm}$ appearing in the NCD films (Figs. 1(d) (f)). The high magnification image (the inset of Fig. 1(d)) shows that many nuclei appear on the profile faces of the grains. Previous literature reported that the homoepitaxial (111) diamond growth can be easily interrupted by re-nucleation under
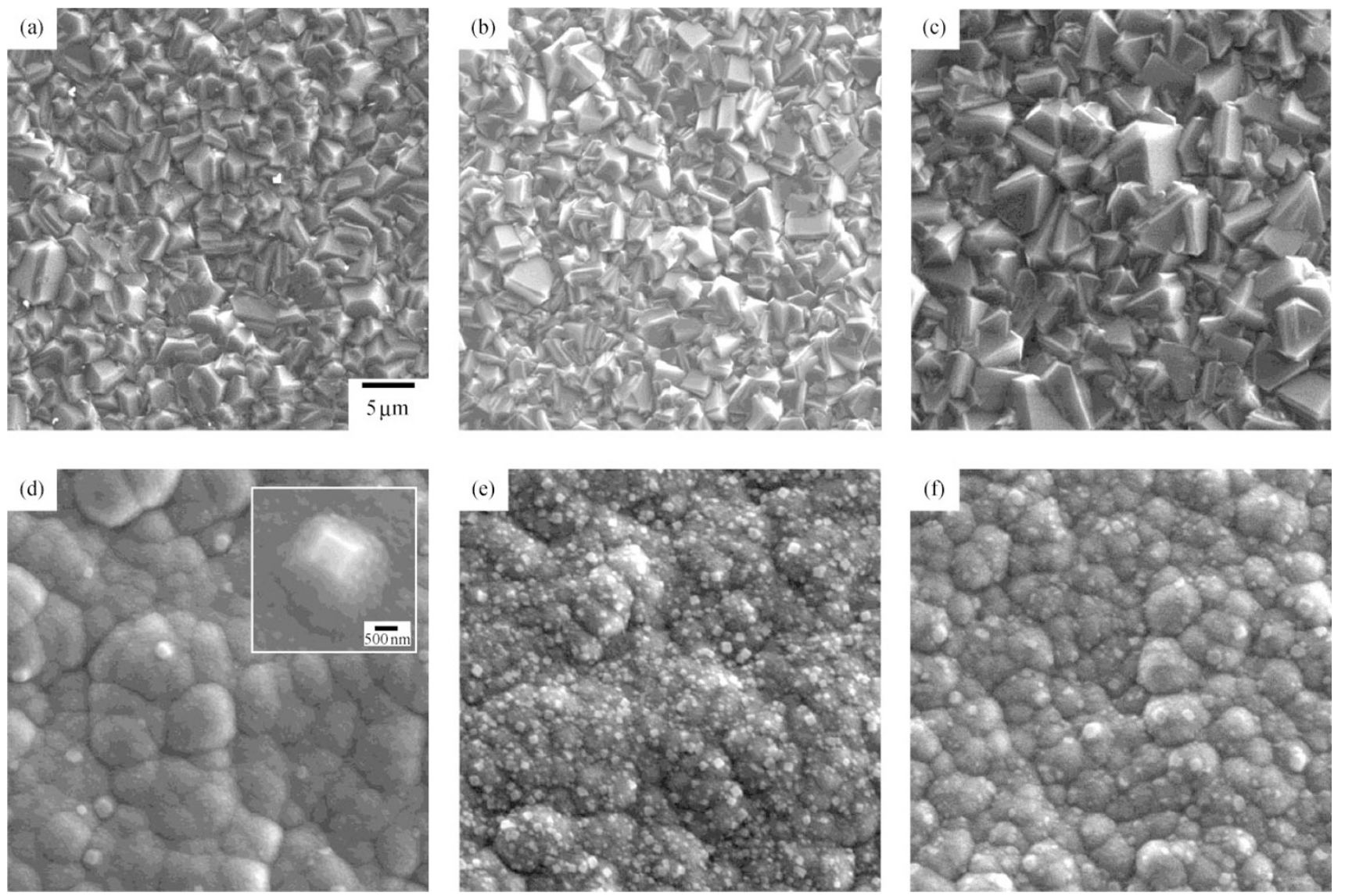

FIG. 1. SEM images of diamond films deposited with various N: B (flow rate in sccm) ratios of 1:2, 4:2, 8:2, 1:10, 4:10 and 8:10, labeled as samples (a) (f) in turn. The scale bars for all images are the same as shown in (a). 
a suitable condition, while the (001) textured layer is more stabilized especially when the [001] axes perpendicular to the substrate [10]. In our case, the abundant re-nucleations cover the non-[001] grains in NCD films. Therefore, the [100] grains are grown with non-[001] grains, which results in the formation of round shaped features.

In the XRD spectra (see Fig. 2) for all the samples, two peaks are generally observed at $43.9^{\circ}$ and $75.2^{\circ}$, which are assigned to the characteristic diamond diffraction patterns of (111) and (220) respectively. Note that the (400) diffraction peak is negligible at high $2 \theta$ angle (not shown), implying that the (100) orientation growth is not evident. In addition, the Si (222) peak appears in the XRD spectra of samples (a) and (d), because the diamond films are thin and the signals from the beneath substrate $\mathrm{Si}$ are evident. It is thus speculated that the diamond films deposited at low nitrogen-flow rates (samples (a) and (d)) generally have low growth rates [4], with respect to the other samples (b), (c), (e) and (f) deposited at high nitrogen-flow rates. The serials MCD films (see Fig. 2A) are mainly [111] texture by comparing the relative intensity ratio of (111) to (220) peak, while the NCD samples (see Fig. 2B) are mainly mixed [110] and [111] texture. For the series of both MCD and NCD films, the intensity ratios of (111) to (220) peak increase with increasing the flow rate of nitrogen, which means that the [111] texture tends to be presented with higher nitrogen concentration introduced. The average grain sizes are 13.2, 12.8 and $15.5 \mathrm{~nm}$ for samples (d)-(f), respectively, estimated from (111) peak by the well-known Scherrer formula:[11] $d=0.9 \lambda /(D \cos \theta)$, where $\lambda$ is $1.54016 \AA, D$ is the full width at half maximum (FWHM). Obviously, the grain size varies insignificantly with the additions of nitrogen for NCD films, which is consist with the observations

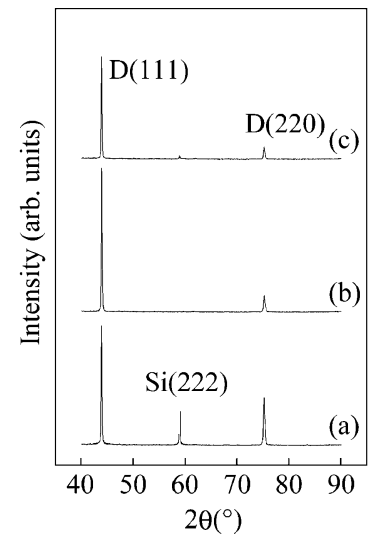

(A)

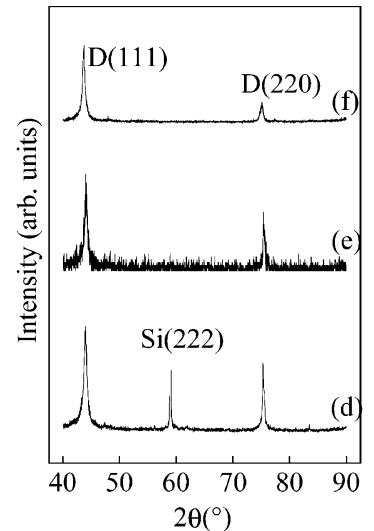

(B)
FIG. 2. XRD spectra of the serial MCD films (A) and NCD films (B) deposited with various $\mathrm{N}$ : $\mathrm{B}$ (flow rate in $\mathrm{sccm}$ ) ratios of 1:2, 4:2, 8:2, 1:10, 4:10, and 8:10, labeled as samples (a) (f) in turn. in the SEM images.

Figure 3 shows Raman spectra obtained from the as-grown samples. It is found that for the MCD samples (see Fig. 3A), the zone-centre phonon band of diamond became asymmetry and downshift to about $1300 \mathrm{~cm}^{-1}$ due to the Fano interference [12]. In addition, two broad bands around 500 and $1200 \mathrm{~cm}^{-1}$ appear in the low frequency part, which are in agreement with the two maxima of phonon density of states of diamond [13,14]. The peak at around $500 \mathrm{~cm}^{-1}$ shows a blue-shift with the increase of the introducing nitrogen, implying that the boron content is reduced [5].

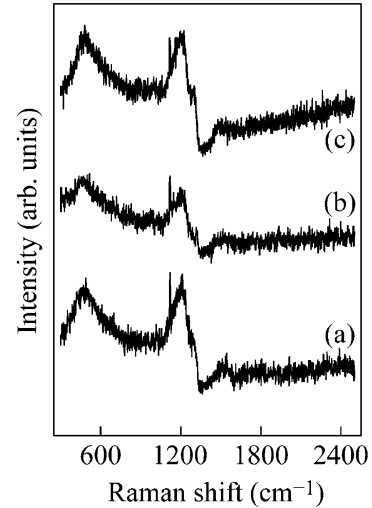

(A)

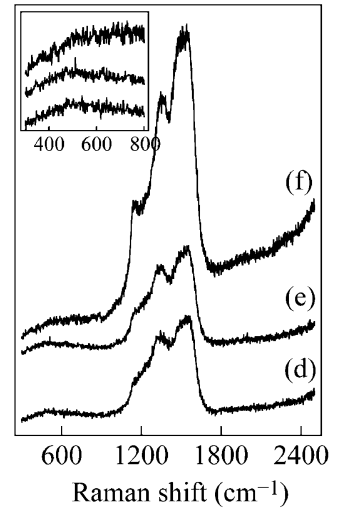

(B)
FIG. 3. Raman spectra of the serial MCD films (A) and NCD films (B) deposited with various $\mathrm{N}$ : $\mathrm{B}$ (flow rate in sccm) ratios of 1:2, 4:2, 8:2, 1:10, 4:10, and 8:10, labeled as samples (a) (f) in turn.

However, the Raman spectra of the NCD films (Fig. 3B) are completely different from that of the MCD films. The spectra show the typical features of the NCD films and mainly consist of five featured peaks at around 500, 1140, 1350, 1480, and 1540 $\mathrm{cm}^{-1}$, while the diamond peak at $\sim 1332 \mathrm{~cm}^{-1}$ is nearly absence. The strong peaks at $\sim 1350 \mathrm{~cm}^{-1}$ and $\sim 1540 \mathrm{~cm}^{-1}$ correspond to the D (disordered carbon) band and G (graphitic carbon) band, respectively. The shoulder peak at $\sim 1140 \mathrm{~cm}^{-1}$ is usually referred to the signature of nanodiamond [15] and/or is accompanied by another peak at $\sim 1480 \mathrm{~cm}^{-1}$ related to the presence of transpolyacetylene (TPA) states in the grain boundaries of NCD films [16]. When the nitrogen concentration increased, the peak around $500 \mathrm{~cm}^{-1}$ decreased continually (inset of Fig. 3B) and the $1140 \mathrm{~cm}^{-1}$ peak became more visible, indicating the incorporation of boron has been prevented and the addition of nitrogen improves the diamond structure, which are consistent with the results reported in refs 17 and 18 .

As previous reported that high concentration of nitrogen and methane was desirable to grow NCD film [19], however, in our experiments the nitrogen and methane were kept constant 
with low concentrations. We further fabricated a series of samples fabricated with a boron flow rate of $5 \mathrm{sccm}$ by adjusting the nitrogen flow rate. The SEM images as shown in Fig. 4 indicted that the features of the deposited films are similar with samples (d) $\sim$ (f) but content more diamond grains. Therefore, the transformation from MCD to NCD is gradually occurred with increasing the boron flow rate, which is related to the existence of more activated boron atoms and $\mathrm{CH}_{\mathrm{x}}$ ions decomposed from $\mathrm{B}\left(\mathrm{OCH}_{3}\right)_{3}$ in the reactive gases. The increase of boron atoms could significantly change the growth ambience to decrease the grain size, and the $\mathrm{CH}_{\mathrm{x}}$ ions will reduce the relative concentration of $\mathrm{H}$ atoms, resulting in the increase of non-diamond phase content and hindrance to the diamond phase growth [20].
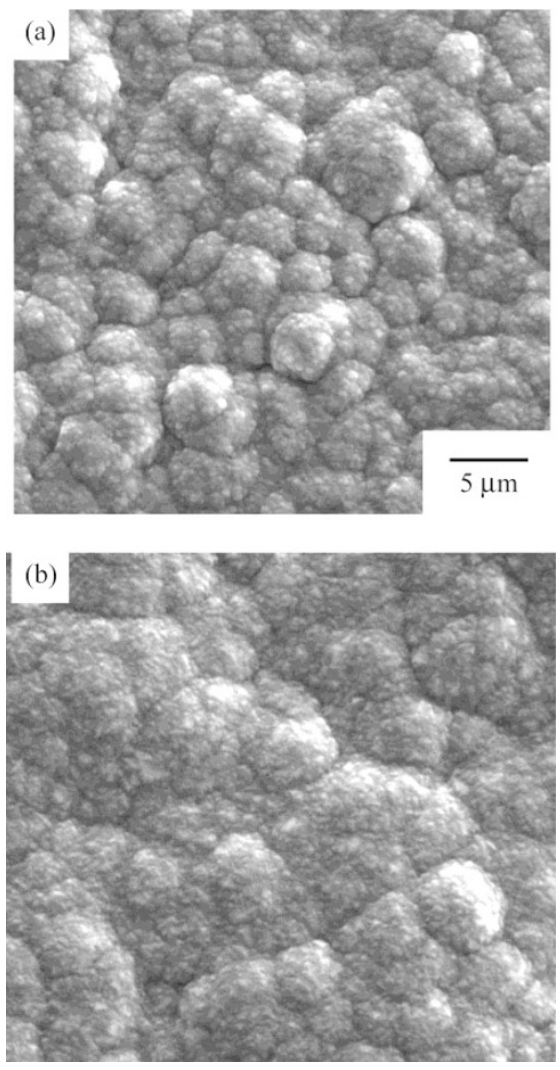

FIG. 4. SEM images of diamond films deposited with N: B (flow rate in sccm) of 4:5 (a) and 8: 5 (b).

The EFE characteristics illustrated as the current density-electric field $(J-E)$ and Fowler-Nordheim $(F-N)$ curves are plotted in Fig. 5 for all the samples. The important parameters for EFE, i.e., turn-on field $\left(E_{0}\right.$, defined as the applied field corresponding to the current density of $0.5 \mu \mathrm{A} / \mathrm{cm}^{2}$ ) and $\mathrm{FE}$ current density $\left(J_{\mathrm{e}}\right)$, are summarily listed in Table 1 . For both MCD and NCD samples, the EFE properties are enhanced (with low $E_{0}$ and high $J_{\mathrm{e}}$ ) at an optimum nitrogen flow rate (i.e., samples (b) and (f)). The F-N plot shows two straight lines in the region of the low and high voltage regions for all the curves,

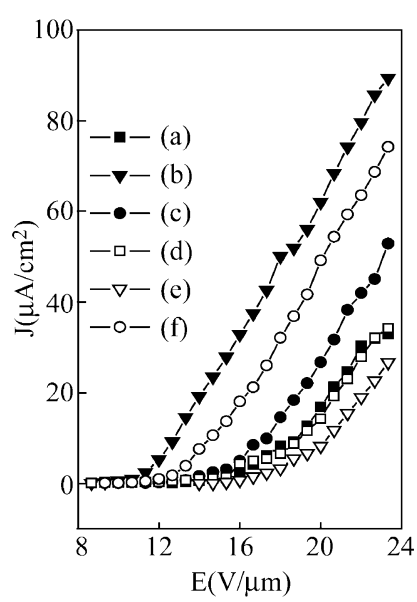

(A)

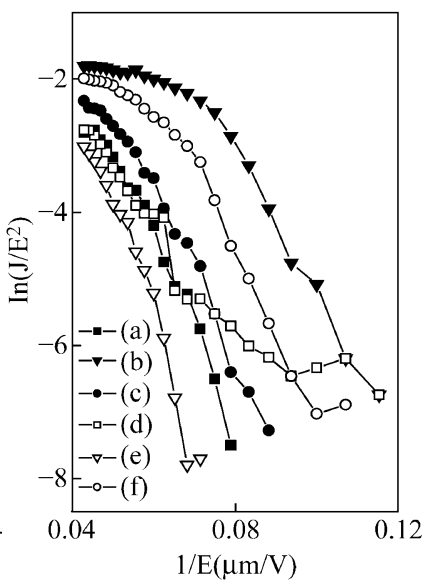

(B)
FIG. 5. Current density-electric field curve (A) and Fowler-Nordheim curve (B) of diamond films deposited with $\mathrm{N}$ : $\mathrm{B}$ (flow rate in sccm) ratios of 1:2, 4:2, 8:2, $1: 10,4: 10$, and 8:10, labeled as samples (a) (f) in turn.

suggesting that electron emission in those samples are due to Fowler-Nordheim tunneling through the surface potential barrier, which is varied at different applied field regions [21]. Note that at nearly the same turn-on field, the current densities in our experiments are smaller than the previous data in the order of $\mathrm{mA} / \mathrm{cm}^{2}$ [9]. The origination is not clear in the current work, and further study is being carried out.

It is known that in $\mathrm{N}$ - and/or B-doped polycrystalline diamond films, a number of $\mathrm{sp}^{2}$-bonded carbon generally appear and trend to accumulate on the grain surface and boundaries [22]. The $\mathrm{sp}^{2}$-bonded phase overcoated between grains can lower down the energy barrier and even additively plays a conductive channel for EFE [23]. Note that too much non-diamond phase would relatively degrade the EFE properties due to the larger work function as compared to diamond [24]. In our experiments, for MCD films, small amount of incorporated nitrogen would

Table 1. The electron field emission properties of the diamond films deposited with various N: B ratios of 1:2, 4:2, 8:2, 1:10, 4:10 and 8:10 in sccm, labeled as samples (a) (f) in turn.

\begin{tabular}{ccccccc}
\hline Sample & $\mathrm{a}$ & $\mathrm{b}$ & $\mathrm{c}$ & $\mathrm{d}$ & $\mathrm{f}$ & 12.7 \\
$E_{0}(\mathrm{~V} / \mu \mathrm{m})$ & 14.0 & 10.0 & 13.3 & 11.3 & & 16.0 \\
$J_{\mathrm{e}}\left(\mu \mathrm{A} / \mathrm{cm}^{2}\right)$ at $E=23 \mathrm{~V} / \mu \mathrm{m}$ & 33.0 & 89.4 & 52.9 & 34.2 & 26.7 \\
\hline
\end{tabular}


introduce $\mathrm{sp}^{2}$-bonded phase, which can enhance the EFE property by reducing the energy barrier (see Fig. 5(A)) [25]. However, the excessive nitrogen reduces the conductivity by preventing the incorporation of boron, leading to the degradation of EFE properties. For the case of NCD films with superabundant non-diamond phase, the increasing incorporation of nitrogen can enhanced diamond component and depress the formation of non-diamond phase (as shown in Raman spectra), which would consequently enhance the EFE property (see Fig. 5(B)).

Furthermore, it should be pointing out that the EFE properties of best MCD film (sample (b) is superior to all the NCD films. For understanding this issue, on the one hand, the incorporation of excess boron species substantially reduces the concentration of dangling bonds that markedly decrease the amount of impurity bands in NCD films [26], which would strongly influence the EFE properties of the products. On the other hand, the [111] texture of MCD films possess much rougher surface than the NCD films, which provides a higher field enhancement factor than the mixed [110] and [111] geometry [27].

In summary, we fabricated boron-doped microcrystalline diamond (MCD) and nanocrystalline diamond (NCD) films by adjusting the gas proportion of nitrogen and boron in the reaction ambient. The XRD results show that the MCD films are of predominated [111] texture, while the NCD films are mixed [110] and [111] texture. The Raman spectra demonstrate that the nitrogen has different effect on the content of non-diamond phase and conductivity for NCD films as compared with MCD films. The discrepancy of EFE characteristics between MCD and $\mathrm{NCD}$ are ascribed to the differences in the content of $\mathrm{sp}^{2}$-bonded carbon, texture feature, and boron concentration in the films. The optimized nitrogen introductions have been realized to improve EFE properties of N- and B-doped CVD diamond films.

This work was financially supported by The Program for New Century Excellent Talents in University (NCET), the National Natural Science Foundation of China (NSFC) under Grant No. 50772041.

Received 7 June 2010; accepted 16 July 2010; published online 20 August 2010.

\section{References}

1. W. Saslow, T. K. Bergstresser and M. L. Cohen, Phys. Rev. Lett. 16, 354 (1966). doi:10.1103/PhysRevLett.16.354.
2. I. N. Lin, K. G. Perng, L. H. Lee, C. F. Shih and K. S. Liu, Appl. Phys. Lett. 77, 1277 (2000). doi:10.1063/1. 1289903.

3. C. J. Tang, A. J. Neves, S. Pereira, A. J. Fernandes, J. Grácio and M C Carmo, Diam. Relat. Mater. 17, 72 (2008). doi:10.1016/j.diamond.2007.10.022.

4. H. D. Li, G. T. Zou, Q. L. Wang, S. H. Cheng, B. Li, J. N. Lv, X. Y. Lv and Z. S. Jin, Chin. Phys. Lett. 25, 1803 (2008). doi:10.1088/0256-307X/25/5/069.

5. L. A. Li, H. D. Li, X. Y. Lu, S. H. Cheng, Q. L. Wang, S. Y. Ren, J. W. Liu and G. T. Zou, Appl. Surf. Sci. 256, 1764 (2010). doi:10.1016/j.apsusc.2009.09.109.

6. D. Lu, H. D. Li, S. H. Cheng, J. J. Yuan and X. Y. Lv, Nano-Micro Lett. 2, 56 (2010). doi:10.1116/1.590618.

7. Q. Hu, M. Hirai and R. K. Joshi, J. Phys. D: Appl. Phys. 42, 025301 (2009). doi:10.1088/0022-3727/42/2/025301.

8. P. Hartmann, R. Haubner and B. Lux, Diam. Relat. Mater. 6, 456 (1997). doi:10.1016/S0925-9635(96)00636-X.

9. I. N. Lin, T Hsu, G. M. Lin, Y. P. Chou, T. T. Chen and H. F.Cheng, J Vac. Sci. Technol. B 21, 1074 (2003). doi:10.1116/1.1576396.

10. X. Jiang, W. J. Zhang, M. Paul and C. P. Klages, Appl. Phys. Lett. 68, 1927 (1996). doi:10.1063/1.115628.

11. P. Scherrer and G. Nachrichten, Kolloidchemie (3rd Ed. 1920), p. 394 (1918).

12. M. Nesládek, D. Tromson, C. Mer, P. Bergonzo, P. Hubik and J. Mares, Appl. Phys. Lett. 88, 232111 (2006). doi:10.1063/1.2211055.

13. S. Jeedigunta, P. Spagnol, J. Bumgarner and A. Kumar, Diam. Relat. Mater. 17, 2037 (2008). doi:10.1016/ j.diamond.2008.06.012.

14. H. D. Li, T. Zhang, L. A. Li, X. Y. Lv, B. Li, Z. S. Jin and G. T. Zou, J. Crys. Growth 312, 1986 (2010). doi:10.1016/ j.diamond.2008.06.012.

15. L. Fayette, B. Marcus, M. Mermoux, G. Tourillon, K. Laffon, P. Parent and F. Normand, Phys. Rev. B 57, 14123 (1998). doi:10.1016/j.diamond.2008.06.012.

16. A. C. Ferrari and J. Robertson, Phys. Rev. B 63 121405-1 (2001). doi:10.1103/PhysRevB.63.121405.

17. Q. Liang, S. A. Catledge and Y. K. Vohra, Appl. Phys. Lett. 83, 5047 (2003). doi:10.1103/PhysRevB.63.121405.

18. R. Locher, J. Wagner, F. Fuchs, C. Wild, P. Hiesinger, P. Gonon and Koidl, Mater. Sci. Eng. B 29, 211 (1995). doi:10.1016/0921-5107(94)04045-6.

19. D. Pradhan, Y. C. Lee, C. W. Pao, W. F. Pong and I. N. Lin, Diam. Relat. Mater. 15, 2001 (2006). doi:10.1016/ 
0921-5107(94)04045-6.

20. Z. L. Wang, C. Lu, J. J. Li and C. Z. Gu, Diam. Relat. Mater. 18, 132 (2009). doi:10.1016/0921-5107(94) 04045-6.

21. S. Q. Li, Y. X. Liang and T. H. Wang, Appl. Phys. Lett. 88, 053107 (2006). doi:10.1016/0921-5107(94)04045-6.

22. P. Zapol, M. Sternberg, L. A. Curtiss, T. Frauenheim and D. M. Gruen, Phys. Rev. B 65, 045403 (2001). doi:10.1016/0921-5107(94)04045-6.

23. G. J. Amaratunga and S. P. Silva, Appl. Phys. Lett. 68, 2529 (1996). doi:10.1063/1.116173.
24. D. Pradhan and I. N. Lin, Appl. Mater. Interfaces 1, 1444 (2009). doi:10.1063/1.116173.

25. D. Zhou, A. R. Krauss, L. C. Qin, T. G. McCauley and D. M. Gruen, J Appl. Phys. 82, 4546 (1997). doi:10.1063/ 1.116173.

26. Y. H. Chen, C. T. Hu and I. N. Lin, Appl. Phys. Lett. 75, 2857 (1999). doi:10.1063/1.125173.

27. H. Ji, Z. S. Jin, J. Y. Wang, X. Y. Lu, C. Z. Gu, B. B. Liu, W. C. Jin and C X Gao, J Vac. Sci. Technol. B 17, 684 (1999). doi:10.1116/1.590618. 